\title{
Papéis Sexuais, Amor e Satisfação Conjugal em Indivíduos Heterossexuais e Homossexuais
}

\author{
José Augusto Evangelho Hernandez' ${ }^{1}$ \\ Vera Lucia da Annunciação Baylão ${ }^{2}$ \\ ${ }^{1}$ Universidade do Estado do Rio de Janeiro (UERJ) \\ ${ }^{2}$ Pontifícia Universidade Católica do Rio de Janeiro
}

\begin{abstract}
Resumo
A experiência do amor entre pessoas do mesmo sexo ainda é percebida numa ótica social estigmatizante. Na tentativa de contribuir com a discussão dessa questão, neste estudo, foram geradas evidências de validade e fidedignidade para o Bem Sex Role Inventory - reduzido por meio da análise dos escores de 331 indivíduos heterossexuais e homossexuais, de ambos os sexos, que coabitavam com seus parceiros amorosos há pelo menos um ano no Rio de Janeiro/RJ. Também foi realizada uma análise confirmatória da Escala Triangular do Amor - reduzida, que revelou bom ajuste do modelo e invariância de medida para homossexuais e heterossexuais. Além disso, foram verificadas as relações entre orientação sexual, papéis sexuais, amor e satisfação conjugal. A intimidade e o compromisso no amor foram os melhores preditores da satisfação conjugal para ambos os grupos. Foram encontradas algumas evidências que questionam os estereótipos sociais vinculados às relações amorosas de pessoas de mesmo sexo.

Palavras-chave: orientação sexual, papéis sexuais, amor, relações conjugais
\end{abstract}

Sex Roles, Love, and Conjugal Satisfaction in Heterosexual and Homosexual Individuals

\begin{abstract}
The experience of same-sex love is still perceived from a stigmatizing social perspective. In an attempt to contribute to the discussion of this issue, this study generated evidence of validity and reliability for the Bem Sex Role Inventory-Reduced by analyzing the scores of 331 heterosexual and homosexual individuals, of both sexes, who cohabited with their partners for at least one year in the city of Rio de Janeiro, state of Rio de Janeiro. A confirmatory analysis of the Triangular Scale of Love-Reduced was also performed, which revealed good model fit and invariance of measurement for homosexuals and heterosexuals. In addition, the relationships between Sexual Orientation, Sex Roles, Love and Conjugal Satisfaction were verified. Intimacy and Commitment were the best predictors of Conjugal Satisfaction for both groups. Some evidence was found questioning the social stereotypes associated to same-sex relationships.

Keywords: sexual orientation, sex role, love, marital relations
\end{abstract}

Roles sexuales, amor y satisfacción conyugal en individuos heterosexuales y homosexuales

\begin{abstract}
Resumen
La relación amorosa entre personas del mismo sexo aún es percibida desde una estigmatizante perspectiva social. Tratando de contribuir con la discusión de este tema, en este estudio se generaron evidencias de validez y fidedignidad para el Bem Sex Role Inventory-Reducido, por medio del análisis de los resultados obtenidos de 331 individuos heterosexuales y homosexuales, de ambos sexos, que viven con sus parejas por lo menos desde hace un año en Río de Janeiro / RJ. También se realizó un análisis confirmatorio de la Escala Triangular del Amor-Reducida, que reveló buen ajuste del modelo e invariancia de medida para homosexuales y heterosexuales. Se verificaron las relaciones entre Orientación Sexual, Roles Sexuales, Amor y Satisfacción Conjugal. La Intimidad y Compromiso en el amor fueron los mejores predictores de Satisfacción Conjugal para ambos grupos. Se encontraron algunas evidencias que cuestionan los estereotipos sociales vinculados a relaciones amorosas entre personas del mismo sexo.

Palabras clave: orientación sexual, rol sexual, amor, relaciones conjugales
\end{abstract}

Os dados publicados sobre os tamanhos das populações de casais homossexuais, em geral, não são confiáveis. No Brasil, o Instituto Brasileiro de Geografia e Estatística, na Pesquisa Nacional por Amostragem de Domicílio, não contempla esse dado ou não faz a coleta de forma precisa (Bergamo, 2016). Nos Estados Unidos, DiBennardo e Gates (2014) sustentaram que as estatísticas que são produzidas pelas pesquisas subestimam o tamanho real da população de casais homossexuais que, na realidade, seria significativamente maior. Gates e Newport (2015), baseados no Gallup Daily, pesquisa diária que avalia as opiniões e percepções, sugeriram que cerca de dois milhões de norte-americanos formam casais de mesmo sexo. Portanto, parece imprescindível que essas relações conjugais sejam também exploradas em pesquisas científicas.

Embora exista, há muitas décadas, a Declaração Universal dos Direitos Humanos, é necessária uma 
discussão sobre os problemas enfrentados pelas minorias sociais na atualidade. A experiência da intimidade romântica entre pessoas do mesmo sexo ainda ocorre em um clima social estigmatizante. As relações sexuais dessas pessoas são, em geral, o ponto focal para a discriminação interpessoal, que tem impacto negativo no bem-estar e na saúde mental dos envolvidos (Frost \& LeBlanc, 2014). A Psicologia pode ajudar a derrubar esses estereótipos negativos, testando teorias sobre os relacionamentos homossexuais e fornecendo bases científicas para a clínica psicológica e as políticas públicas (Lafontaine, Gabbay, Péloquin, Flesch, \& Fitzpatrick, 2013). É nessa linha de ação que a presente pesquisa buscou dar sua contribuição.

Até os anos 60, os pesquisadores da Psicologia procuraram avaliar os aspectos masculinos e femininos das pessoas baseados em um modelo bipolar excludente. $\mathrm{O}$ paradigma central era o pressuposto de que homens e mulheres diferiam nos estereótipos sociais, nos padrões de desempenho de papéis sexuais e nas prescrições culturalmente aprovadas para os sexos. Contrapondo-se a esse modelo, as Teorias Cognitivas de Processamento de Informações elaboraram modelos explicativos do desenvolvimento e funcionamento desses estereótipos sociais com base em esquemas cognitivos integráveis ao autoconceito (Liben \& Bigler, 2017).

$\mathrm{Na}$ Gender Schema Theory, os indivíduos podem ser classificados como esquemáticos ou não esquemáticos. Em um polo, estariam situados os sujeitos esquemáticos, tipificados sexuais (fortes em masculinidade ou em feminilidade) e, no polo oposto, os não esquemáticos, andróginos (fortes em masculinidade e em feminilidade) e os indiferenciados (fracos em masculinidade e em feminilidade). Essa classificação baseou-se nas diferenças individuais quanto aos recursos cognitivos disposicionais, tanto em nível de discriminação perceptiva, associativa e de memória, quanto no que se refere às expectativas e crenças sobre a polaridade de gêneros (Starr \& Zurbriggen, 2017).

Uma vez aprendido, o esquema de gênero predisporia o indivíduo a perceber o mundo em termos sexuais e serviria para a pessoa avaliar a si própria e aos que a rodeiam quanto à adequação aos padrões definidos pela sociedade para os sexos (Starr \& Zurbriggen, 2017). Os esquemáticos teriam maior predisposição para classificar as informações nas categorias masculina e feminina tradicionais e para decidir quais atributos incluiriam ou não em seu autoconceito. Quando o indivíduo, percebesse a sua própria conformidade ao padrão tradicional, a diferenciação do autoconceito baseada em distinções de sexo seria fortalecida e resultaria em uma identidade de papel sexual convencional. Os tipificados sexuais tenderiam a dar mais atenção às estratégias de coping apropriadas aos sexos, desconsiderando as mudanças do ambiente. Por outro lado, os andróginos não contariam com conotações sexuais para orientar o seu processamento de informações, o que questionaria a suposição de que a masculinidade é o oposto da feminilidade e defenderia que as pessoas podem ter esses atributos simultaneamente (Dean \& Tate, 2017).

$\mathrm{Na}$ pesquisa sobre relacionamentos íntimos, a Teoria Triangular do Amor de Sternberg (2006) sustentou que o amor compreende três componentes: paixão, intimidade e decisão/compromisso. Sternberg revisou a literatura norte-americana e de outros países e constatou que, embora esses componentes não tenham o mesmo peso em todas as culturas, no mínimo, têm algum peso e se manifestam no amor em qualquer época ou lugar.

A Paixão seria o elemento romântico e físico, a sensação romântica, a atração física, o desejo de estar junto, a satisfação e o contentamento sexual mútuo e a excitação física e emocional. $\mathrm{Na}$ Intimidade, está o desejo de promover o bem-estar de outra pessoa, a felicidade de poder compartilhar experiências de vida, o senso de respeito mútuo, a capacidade de contar com a pessoa amada em momentos de necessidade, a compreensão mútua nos momentos difíceis, a compreensão íntima compartilhada, a disposição de dar e receber apoio emocional da pessoa amada, a capacidade de se comunicar além dos níveis superficiais e o reconhecimento mútuo do valor do parceiro. A Decisão/Compromisso teria duas fases: a decisão de que amamos alguém e o compromisso, a determinação de manter esse amor para sempre. Esse componente compreenderia a certeza de que o sentimento do indivíduo é um tipo especial de amor, a disposição para simbolizar ou articular isso de algum modo, a compreensão de que o relacionamento é mais do que um capricho passageiro e a determinação de investir nele (Sternberg, 2006). Sternberg (1997) apresentou as propriedades psicométricas da Escala Triangular do Amor (ETAS) em estudo que gerou evidências de validade para o seu modelo teórico.

Os estudiosos têm compreendido a satisfação conjugal como abrangendo sentimentos subjetivos dos parceiros nos relacionamentos. Esta tem sido definida como o grau em que um indivíduo está satisfeito com sua relação romântica e tem sido considerada a 
melhor preditora da estabilidade do amor (Kochar \& Sharma, 2015).

Níveis maiores de satisfação conjugal foram relatados por casais de lésbicas em comparação com gays e heterossexuais. Foi sugerido que essas experiências das lésbicas possam estar associadas com suas maneiras positivas de lidar com as dificuldades da relação, demonstradas por maior expressão de afeto (Kurdek, 2008). Porém, na pesquisa de Hernandez e Soares (2013), a Satisfação Conjugal observada nas lésbicas foi menor do que a dos gays e heterossexuais. Contudo, é preciso considerar que esse estudo abordou três casos e o casal de lésbicas, embora mantivesse relação há vários anos, não coabitava. Por outro lado, várias pesquisas não têm encontrado relação entre Orientação Sexual e Satisfação Conjugal (Gottman et al., 2003; Kurdek, 2006; Means-Christensen, Snyder \& Negy, 2003).

Considerando as relações entre os papéis sexuais e a satisfação conjugal, os casais esquemáticos poderiam ter mais dificuldades nos relacionamentos (Bailey, Hendrick, \& Hendrick, 1987). Os homens que são avaliados como influenciados fortemente por normas tradicionais de gênero têm sido associados negativamente com a Satisfação Conjugal. Esse efeito apareceu tanto nos autorrelatos desses homens tradicionais quanto nas avaliações de suas mulheres (Burn \& Ward, 2005). De outra forma, as pessoas andróginas tenderiam a ser mais flexíveis, saudáveis e com maior capacidade para amar, pois seriam mais conscientes, expressivas e tolerantes (Yela, 1998). Nos resultados de Curun (2012), os heterossexuais que perceberam seus parceiros como andróginos apresentaram maiores níveis de satisfação na relação do que quando perceberam seus parceiros com outros tipos de papéis sexuais, porém essa amostra era de pessoas envolvidas em um relacionamento do tipo namoro.

No estudo atual, foram produzidas evidências de validade e fidedignidade para as versões reduzidas do BSRI e da ETAS e examinadas as relações entre as autopercepções de papel sexual, amor e satisfação conjugal de indivíduos héteros e homossexuais. Assim, buscou-se contribuir com subsídios para a clínica psicológica de casais e para o desenvolvimento de políticas públicas social e de saúde.

\section{Método}

\section{Participantes}

Foram examinadas 331 pessoas, 157 (47,4\%) do sexo masculino, sendo $85(54,1 \%)$ heterossexuais e 72
$(45,9 \%)$ homossexuais; e, $174(52,6 \%)$ do sexo feminino, sendo $92(52,9 \%)$ heterossexuais e $82(47,1 \%)$ homossexuais do Estado do Rio de Janeiro/Brasil. A idade dos participantes variou de 18 a 78 anos $(M$ $=36,3 ; D P=10,56)$. Todos os participantes estavam em algum tipo de relacionamento amoroso e, necessariamente, coabitavam com os parceiros. O tempo de duração dessas relações variou de um a 46 anos $(M=$ $8,2 ; D P=8,30)$.

\section{Instrumentos}

$\mathrm{Na}$ validação da teoria, Bem (1974) construiu o Bem Sex Role Inventory (BSRI), que foi projetado para autoavaliar os traços de personalidade de gênero. A escala masculina contém 20 adjetivos estereotipados associados aos homens (tais como valente, autoconfiante etc.) e a escala feminina, 20 adjetivos associados às mulheres (tais como delicada, graciosa etc.). Para este estudo, foi produzida uma versão reduzida (BSRI-R) ad hoc de 15 itens a partir da adaptação brasileira do BSRI (Hernandez, 2009). Foram selecionados itens que apresentaram carga fatorial $>0,50$ nos fatores para os quais foram designados: cinco para a escala de masculinidade, cinco para feminilidade e cinco para uma escala neutra de características indesejáveis. Os respondentes usaram uma escala tipo Likert de sete pontos (1, "a característica nunca é verdadeira" e 7, "a característica sempre é verdadeira”) para avaliar os adjetivos em relação às suas próprias características pessoais. O ponto de corte para a determinação dos papéis sexuais de cada um foi estabelecido pelo cálculo da mediana das médias das escalas de masculinidade e feminilidade, método estabelecido por Sandra Bem. Participantes com médias acima do ponto de corte em masculinidade e abaixo em feminilidade foram classificados como Típicos Masculinos; no inverso, foram classificados como Típicos Femininos. Os indivíduos com médias acima do ponto de corte em masculinidade e feminilidade foram classificados como Andróginos e, com médias abaixo, foram rotulados como Indiferenciados.

Para validar sua teoria, Sternberg (1997) construiu a Escala Triangular do Amor (ETAS) com 45 itens distribuídos igualmente entre os três componentes do amor. O estudo forneceu algumas evidências de validade de construto para a ETAS, mas revelou problemas para o modelo testado: elevados índices de correlação entre fatores e cross-loads de itens.

Nas adaptações brasileiras da ETAS, persistiram esses problemas e, na tentativa de superá-los, Gouveia, Fonseca, Cavalcanti, Diniz e Dória (2009) definiram 
uma versão reduzida (ETAS-R) com 15 itens, igualmente divididos entre Paixão, Intimidade e Decisão/ Compromisso. Nesta pesquisa, para responder a ETAS-R, os sujeitos usaram uma escala tipo Likert de sete pontos, onde 1 é "nada verdadeiro", 4 é "moderadamente verdadeiro" e 7 é "totalmente verdadeiro". No estudo de Hernandez (2016), a ETAS-R mostrou-se competente na representação do seu construto, seus alfas variaram de 0,80 a 0,92 .

Para avaliar a Satisfação Conjugal foi usada a Escala de Avaliação do Relacionamento (EAR) de Hendrick (1988), uma escala unidimensional de sete itens que fornece uma medida global. Os itens são frases interrogativas que foram avaliadas a satisfação na relação por meio de uma escala tipo Likert de cinco pontos, onde 1 é "nada" e 5 "totalmente". Na adaptação brasileira, foram reveladas evidências de validade de construto para a EAR e o alfa foi 0,87 (Hernandez, 2014). Além desses dados, foram coletadas informações sobre o sexo, a idade, a orientação sexual e o tempo de duração da relação amorosa.

\section{Procedimentos}

Este projeto foi submetido e aprovado pelo Comitê de Ética da instituição que está vinculado. Os participantes foram convidados e informados sobre os objetivos da pesquisa, leram e assinaram um Termo de Consentimento Livre e Esclarecido. Os dados foram coletados em diversos ambientes: no campus universitário, nas residências, em igrejas, em paradas gays, em encontros científicos e em entidades da diversidade sexual. Em todos os casos, os participantes foram abordados e, de forma individual, responderam aos questionários na presença dos pesquisadores.

\section{Análise dos Dados}

A versão do BSRI-R foi submetida à análise fatorial exploratória (AFE), no software Factor, por meio da matriz de correlação policórica com o método de extração Minimum Rank Factor Analysis (MRFA) e rotação Promin. Para a tomada de decisão de retenção de fatores, foi usada a análise paralela baseada em MRFA.

No âmbito da Modelagem de Equações Estruturais (MEE), foi realizada uma análise fatorial confirmatória multigrupos (AFCMG) para verificar a invariância da estrutura do modelo da ETAS-R entre os grupos de orientação sexual. Foram calculados os seguintes índices de ajustamento: qui-quadrado $\left(\chi^{2}\right)$, que avalia a magnitude da discrepância entre a matriz de covariância populacional e a matriz de covariância da amostra. O $\chi^{2}$ é uma estimativa conservadora do ajuste do modelo quando o tamanho da amostra é > 200 (Byrne, 2016). Nesse caso, deve ser usada a razão $\chi^{2} / g l$ e os resultados $<$ 2,0-3,0 são considerados bons (Arbuckle, 2014); o Comparative Fit Index (CFI), índice que compara o ajuste do modelo avaliado com o modelo basal, valores $>$ 0,90 indicam um bom ajustamento (Marôco, 2014); Parsimony CFI (PCFI), índice de parcimônia, valores entre 6,0-8,0 representam um bom ajuste (Marôco, 2014); o Root Mean Square Error of Approximation (RMSEA), que mede a discrepância por meio dos graus de liberdade entre as estimativas da amostra e da população. Valores $<0,05$ são considerados muito bons (Arbuckle, 2014). Também foram calculadas as variâncias médias explicadas (VME) e as confiabilidades compostas (CC) para as dimensões específicas e global da ETAS-R.

Também foi produzida uma série de análises de regressão múltipla, método Stepwise, para identificar as variáveis preditoras da Satisfação Conjugal para homossexuais e heterossexuais e para os oito grupos resultantes da combinação de orientação sexual e papéis sexuais. As variáveis examinadas foram Idade, Tempo de Relação, Masculinidade, Feminilidade, Paixão, Intimidade e Compromisso (explicativas) e a Satisfação Conjugal (critério). Observe que as variáveis Masculinidade e Feminilidade usadas aqui são intervalares, ou seja, são os escores obtidos no BSRI-R antes da transformação em variáveis categóricas (papéis sexuais). Além disso, análises de variância univariadas compararam os escores médios dos grupos Sexo, Orientação Sexual e Papel Sexual $(2 \times 2 \times 4)$ em Satisfação Conjugal.

\section{Resultados}

Do total de escores do BSRI-R, 18 (0,3\%) estavam ausentes e foram repostos pela média da série. A distribuição multivariada dos escores não era normal, o índice de Mardia foi 174,64 (CR = 47,98), mas, na estatística univariada, a assimetria foi $< \pm 2$ e a curtose $<$ \pm 2 , o que não é numa violação extrema da normalidade (Finney \& DiStefano, 2006). A adequação dos dados à AFE foi verificada por meio do teste $\mathrm{KMO}$, que forneceu um valor de 0,79 , e do teste de esfericidade de Bartlett, que apresentou os valores, $\chi^{2}(153)=1490,60$, $p<0,001$. A análise paralela recomendou a retenção de três fatores (Tabela 1$)$.

Os três fatores extraídos a partir da matriz de correlação policórica revelaram variância comum total de 10,7, e explicada de 7,6 (71,5\%). Os quinze itens do BSRI-R saturaram mais fortes nos fatores designados 
e foram estimados bons índices de fidedignidade para as três escalas (Tabela 2). Na distribuição dos resíduos, o Root Mean Square of Residuals (RMSR) foi de 0,039, menor do que o critério de Kelley para um modelo aceitável $(0,055)$.

Por meio do método de Bem (1974), os respondentes do BSRI-R foram classificados nos Papéis
Sexuais. O Teste do Quiquadrado não apurou associações estatísticas significativas entre Orientação Sexual e Papéis Sexuais, $\chi^{2}(3)=2.955, p=0,399$.

Nos escores da ETAS-R, três estavam ausentes $(0,06 \%$ do total) e foram repostos pela média. A distribuição dos escores da ETAS-R apresentou moderadas assimetrias negativas. Os escores das amostras de

Tabela 1.

Análise Paralela baseada em MRFA

\begin{tabular}{cccc}
\hline Variáveis & \multicolumn{3}{c}{ \% de variância } \\
\hline & Dados Atuais & Média Aleatória & $95^{\circ}$ Percentil Aleatório \\
2 & 24,3 & 13,4 & 15,2 \\
3 & 17,4 & 12,2 & 13,7 \\
4 & 14,9 & 11,2 & 12,4 \\
& 6,6 & 10,2 & 11,2 \\
\hline
\end{tabular}

Nota. Número de 500 matrizes aleatórias de correlações policóricas. Método permutação de dados brutos.

Tabela 2.

Matriz de Cargas Fatoriais das Escalas do BSRI-R

\begin{tabular}{lcccc}
\hline & Feminilidade & Indesejabilidade & Masculinidade & $h^{2}$ \\
\hline Influente & 0,15 & 0,07 & $\mathbf{0 , 5 9}$ & 0,60 \\
Doce & $\mathbf{0 , 6 9}$ & $-0,07$ & 0,01 & 0,59 \\
Dócil & $\mathbf{0 , 7 3}$ & 0,02 & $-0,01$ & 0,63 \\
Negligente & 0,09 & $\mathbf{0 , 6 8}$ & 0,06 & 0,62 \\
Arrojada(o) & 0,06 & 0,06 & $\mathbf{0 , 4 8}$ & 0,36 \\
Cínica(o) & $-0,07$ & $\mathbf{0 , 6 8}$ & 0,06 & 0,61 \\
Fofoqueira(o) & $-0,01$ & $\mathbf{0 , 7 6}$ & $-0,07$ & 0,72 \\
Competidor(a) & 0,01 & 0,21 & $\mathbf{0 , 5 3}$ & 0,57 \\
Suave & $\mathbf{0 , 7 1}$ & 0,01 & 0,10 & 0,60 \\
Graciosa(o) & $\mathbf{0 , 6 4}$ & 0,03 & 0,09 & 0,53 \\
Leviana(o) & 0,12 & $\mathbf{0 , 7 1}$ & $-0,09$ & 0,61 \\
Líder & $-0,01$ & $-0,02$ & $\mathbf{0 , 6 7}$ & 0,53 \\
Delicada(o) & $\mathbf{0 , 6 8}$ & 0,03 & $-0,08$ & 0,58 \\
Grosseira(o) & $-0,22$ & $\mathbf{0 , 4 1}$ & 0,24 & 0,37 \\
Autoconfiante & 0,07 & $-0,24$ & $\mathbf{0 , 5 1}$ & 0,57 \\
Variância comum explicada & $2,5(29,5 \%)$ & $2,3(27 \%)$ & $\mathbf{1 , 7}(19,8 \%)$ & 0,92 \\
Fidedignidade estimada & 0,95 & 0,88 & & - \\
Correlações & - & $-0,18$ & - & \\
Feminilidade & 0,04 & $-0,07$ & & \\
Masculinidade & & & & \\
\hline
\end{tabular}

Nota. Método de extração: Minimum Rank Factor Analysis. Método de rotação: Promin. 
indivíduos heterossexuais $(n=177)$ e homossexuais $(n$ = 154) foram submetidos à AFCMG, no ambiente da Modelagem de Equações Estruturais, com o método de estimação Máxima Verossimilhança (Figura 1), que se mostra robusto mesmo com uma distribuição não normal de dados (Marôco, 2014).

Conforme os índices de ajustamento obtidos $\left(\chi^{2} / g l=2,16 ;\right.$ CFI $=0,923 ;$ PCFI $=0,76$; RMSEA $=$ 0,059; IC $=90 \%$ ]0,051; 0,068D, o modelo fatorial livre avaliado apresentou um bom ajustamento simultâneo para heterossexuais e homossexuais, indicando invariância configuracional. $\mathrm{Na}$ continuidade, a verificação da invariância da medida (pesos fatoriais iguais) também obteve um bom ajuste para as Orientações Sexuais, $\left(\chi^{2} / g l=2,12\right.$; CFI $=0,920$; PCFI $=0,81$; RMSEA $=0,058$; IC $=90 \%$ ]0,050; 0,066D. A comparação entre os ajustes do modelo livre e do modelo de pesos fatoriais mostrou a invariância métrica entre os grupos, $\mathrm{GL}=12, \mathrm{CMIN}=18.107 ; p=0,112$. Como os valores do $\Delta \chi^{2}$ são sensíveis a pequenas diferenças e influenciados pelo tamanho da amostra, utilizou-se o $\Delta$ CFI $(<0,01)$, que também é um indicador da invariância do modelo (Damásio, 2013). Conforme $\Delta$ CFI $=0,923-0,920=0,003$, o teste corroborou a invariância do modelo para Orientação Sexual.

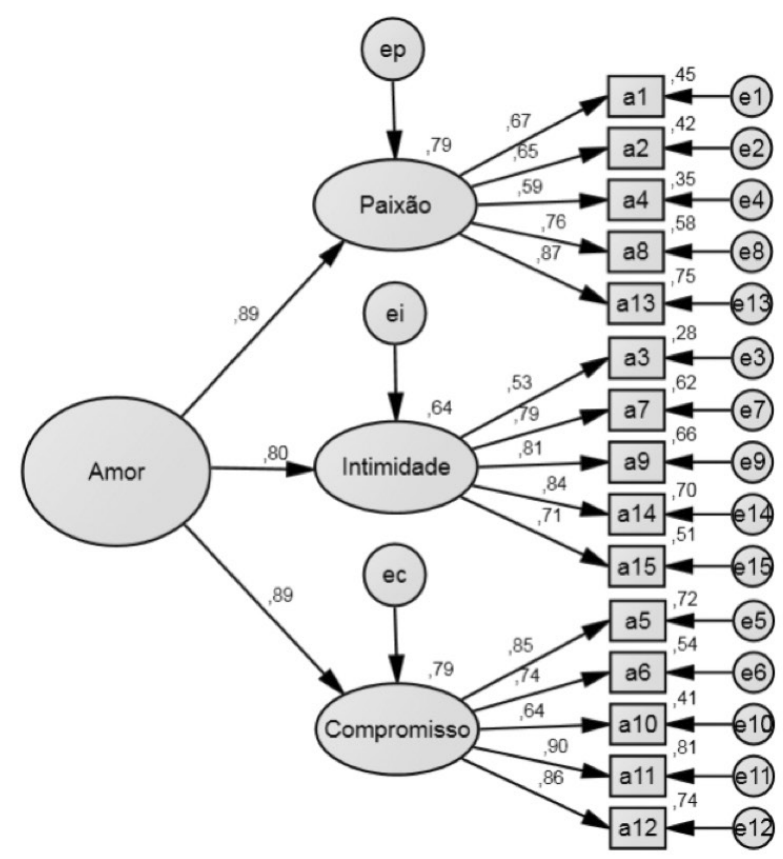

A validade convergente foi medida pela quantidade total das variâncias das variáveis observadas, explicadas pelos fatores latentes para os grupos hétero e homossexual (Tabela 3). A validade discriminante foi verificada pelo método de comparação do quadrado das correlações entre os três fatores da ETAS-R com as VMEs dos mesmos fatores (Fornell \& Larcker, 1981). Em todas as comparações, os valores das VMEs foram maiores do que o quadrado das correlações dos fatores, indicando discriminação entre eles (Tabela 3).

A fidedignidade dos fatores e da dimensão global ETAS-R foi calculada por meio da CC. Foram estimados valores de consistências internas que representam condições adequadas para as dimensões da medida, específicas e global (Tabela 3).

Nas análises de regressões múltiplas, método Stepwise, para cada um dos grupos de Orientação Sexual e Papéis Sexuais $(2 \times 4)$, as variáveis explicativas foram a Paixão, a Intimidade e a Decisão/Compromisso e a variável critério, a Satisfação Conjugal (Tabela 4). No exame da matriz de correlação, as variáveis Sexo, Idade e Tempo de Relação foram excluídas, pois não apresentaram associações estatísticas significativas $(p<$ $0,05)$ com a variável critério. Por outro lado, a Paixão, a Intimidade e a Decisão/Compromisso mostraram correlações significativas com a variável critério e em graus

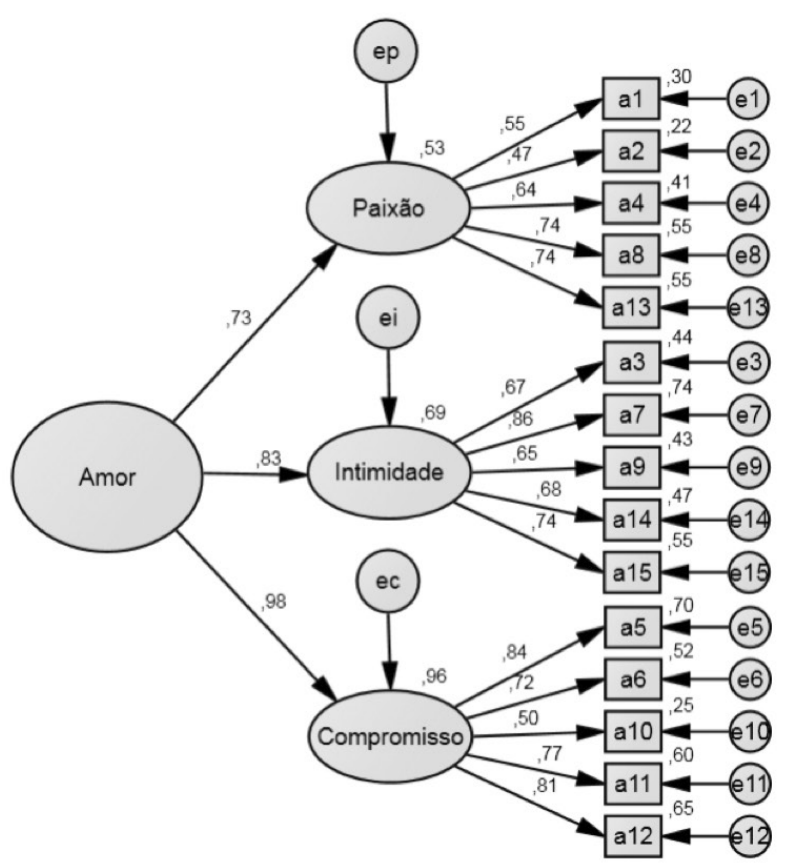

Figura 1. Diagramas da AFCMG com parâmetros livres do modelo da ETAS-R para hetero e homossexuais, respectivamente, com as estimativas padronizadas. 
moderados entre si mesmas $(r<0,70)$, o que caracteriza colinearidade aceitável. Na Tabela 4, a medida de multicolinearidade representada pelo Fator de Inflação da Variância (VIF) também apresentou valores adequados (Hair, Black, Babin, \& Anderson, 2014).

O Teste de Levene, $F(15,315)=922, p=540$, revelou a homogeneidade de variância da Satisfação Conjugal entre os grupos testados. Na ANOVA univariada não foram encontrados efeitos interativos significativos das variáveis Orientação Sexual, Sexo e Papel Sexual, $F(3,315)=508, p=0,677, \eta_{p}^{2}=0,005$, das variáveis Sexo e Orientação Sexual $(2 \times 2), F(1,315)$ $=1.736, p=0,189, \eta_{p}^{2}=0,005$, das variáveis Sexo e Papel Sexual $(2 \times 4), F(3,315)=1.145, p=0,331, \eta_{p}^{2}=$ 0,011, das variáveis Orientação Sexual e Papel Sexual (2 x 4), $F(3,315)=1.161, p=0,325, \eta_{p}^{2}=0,011$, sobre a Satisfação Conjugal. Também não foram significativos os efeitos principais do Sexo, $F(1,315)=2.713$, $p=$ $0,101, \eta_{p}^{2}=0,009$ e do Papel Sexual, $F(3,315)=1.376$, $p=0,250, \eta_{p}^{2}=0,013$ sobre a Satisfação Conjugal. Mas a Orientação Sexual apresentou efeito principal significativo sobre a Satisfação Conjugal, $F(1,315)=6.030, p$ $<0,01, \eta_{p}^{2}=0,019$, embora o tamanho do efeito fosse bastante pequeno. O grupo homossexual teve escores médios $(M=4,1 ; D P=0,53)>$ os do grupo heterossexual $(M=3,9 ; D P=0,63)$.

\section{Discussão}

A AFE com os escores de participantes homo e heterossexuais apresentou evidências de validade fatorial e consistência interna para o BSRI-R, sugerindo que a medida tem três escalas independentes com itens representando, nitidamente, as dimensões para as quais foram designados. O pressuposto fundamental dos estereótipos sociais de gênero de homens e mulheres heterossexuais é que eles devem estar em conformidade com os papéis de gênero tradicionais designados para ambos os sexos. Em geral, a expectativa social estereotipada é de que as mulheres heterossexuais sejam mais

Tabela 3.

Coeficiente de Correlação ao Quadrado, Variância Média Extraída (VME) e Confiabilidade Composta (CC) das Dimensões da ETAS-R para Hetero e Homossexuais

\begin{tabular}{lcccccccc}
\hline & \multicolumn{3}{c}{ Heterossexual } & \multicolumn{3}{c}{ Homossexual } \\
\hline Correlações & F1 & F2 & F3 & ETAS-R & F1 & F2 & F3 & ETAS-R \\
F1-Paixão & - & & & & - & & & \\
F2-Intimidade & 0,32 & - & & & 0,21 & - & & \\
F3-Compromisso & 0,41 & 0,40 & - & & 0,26 & 0,48 & - & \\
VME & 0,51 & 0,55 & 0,64 & & 0,40 & 0,52 & 0,54 & \\
CC & 0,83 & 0,86 & 0,90 & 0,89 & 0,77 & 0,84 & 0,85 & 0,89 \\
\hline
\end{tabular}

Tabela 4.

Regressões Múltiplas das Variáveis Paixão, Intimidade e Compromisso (explicativas) e Satisfação na Relação (critério) para Homo e Heterossexuais

\begin{tabular}{lcccccc}
\hline & $n$ & Modelo & $\mathrm{R}_{\text {Ajustado }}^{2}$ & $\mathrm{~B}$ & $Z(g l)$ & VIF \\
\hline Homossexuais & 154 & 1 Intimidade & 0,41 & 0,64 & $105,649(1,152)^{*}$ & 1,9 \\
& & 2 Intimidade & 0,46 & 0,41 & $67,236(2,151)^{*}$ & \\
& Compromisso & & 0,34 & & \\
Heterossexuais & \multirow{2}{*}{177} & 1 Compromisso & 0,49 & 0,70 & $168,537(1,175)^{*}$ & 1,6 \\
& & 2 Compromisso & 0,57 & 0,47 & $116,233(2,174)^{*}$ & \\
& Intimidade & & 0,37 & & \\
& & & &
\end{tabular}

Nota. ${ }^{*} p<0,001$. 
femininas e menos masculinas do que lésbicas e homens heterossexuais, mais masculinos e menos femininos do que gays. As lésbicas são vistas pela sociedade preconceituosa, de forma generalizada, com masculinidade excessiva e desinteresse pelas aparências e atividades femininas, enquanto os gays, como gentis, passivos e efeminados (Blashill \& Powlishta, 2009).

Contrário às expectativas estereotipadas, nos resultados da pesquisa atual, a análise de distribuição de frequência dos Papéis Sexuais mostrou que os grupos de Orientação Sexual não diferiram significativamente entre si, isto é, os atributos estereotipados não se mostraram associados às Orientações Sexuais. Essa é uma evidência de que o desenvolvimento de um Papel Sexual esquemático ou não esquemático independe de Orientação Sexual. Kachel, Steffens e Niedlich (2016) também não encontraram diferenças estatísticas significativas entre os estereótipos de masculinidade e feminilidade do BSRI e a Orientação Sexual dos participantes de sua pesquisa.

$\mathrm{Na}$ presente pesquisa, os modelos da ETAS-R tiveram bons ajustes e indicativos de invariância para as amostras homo e heterossexual na AFCMG. Os modelos com pesos fatoriais fixos e com covariâncias fixadas entre os fatores e os erros não apresentaram um ajustamento significativamente pior do que o modelo livre, ficando assim demonstrada a invariância de medida do modelo de amor entre homossexuais e heterossexuais. Essa foi uma evidência de igualdade de percepção do construto amor entre as Orientações Sexuais. Baumeister et al., (2011) também testaram o construto do amor de Sternberg e encontraram evidências de adequação dele para jovens gays, semelhante aos estudos anteriores com amostras heterossexuais.

Lewis (2011) encontrou que Intimidade, Paixão e Compromisso são fortes preditores de Satisfação Conjugal em casais heterossexuais. Nos resultados atuais, o Compromisso, com a contribuição menor da Intimidade, foi o principal responsável pela variância da Satisfação Conjugal nas relações heterossexuais. O Compromisso é um elemento crucial na evolução e na manutenção do amor romântico, pois representa a dimensão cognitiva do sentimento de amar, a preferência de estar envolvido e a determinação de continuar a relação (Sternberg, 2006).

A Intimidade, com a participação menor do Compromisso, foi a principal responsável pela variância da Satisfação Conjugal de lésbicas e gays. A Intimidade e seus aspectos tiveram a mesma importância em outros estudos com lésbicas (Lavner, 2017) e com gays
(Deenen, Gijs, \& Naerssen, 1994). A Intimidade é composta por sentimentos de proximidade, de conexão e de vínculo nas relações amorosas. É um investimento emocional e refere-se à promoção do bem-estar do parceiro, à alta consideração, à experiência da alegria, à troca de apoio emocional, à compreensão recíproca, à valorização mútua, ao compartilhamento de pertences e informações pessoais profundas e à comunicação (Sternberg, 2006). Nesses resultados, a Paixão foi excluída dos modelos de predição da Satisfação Conjugal. Esses aspectos conjuntos contradizem o preconceito social de que, nas relações amorosas entre pessoas de mesmo sexo, predominaria o erotismo, a imoralidade e, até, a promiscuidade (Doyle \& Molix, 2015; Farias, 2010; Frost \& Gola, 2015; Peplau \& Fingerhut, 2007; Toledo \& Teixeira Filho, 2012).

Independentemente de Orientação Sexual, no presente estudo não foram encontradas diferenças entre homens e mulheres em Satisfação Conjugal, mas esse tem sido um resultado tradicionalmente controverso nessa linha de pesquisa. A meta-análise de Jackson, Miller, Oka e Henry (2014) indicou que, em média, as mulheres relataram menos Satisfação Conjugal do que os homens, embora o tamanho do efeito tenha sido pequeno e, com a retirada das amostras clínicas, tenha ficado mais reduzido. Rostami, Ghazinour, Nygren e Richter (2014) também encontraram Satisfação Conjugal mais baixa para mulheres na comparação com homens.

No estudo atual, não foram encontradas diferenças estatísticas significativas em Satisfação Conjugal entre os Papéis Sexuais autopercebidos (típico feminino, típico masculino, andrógino e indiferenciado) pelos participantes. Curun (2012), com casais heterossexuais de namorados universitários, também não encontrou diferenças entre as autopercepções de Papéis Sexuais. Mas, nas heteropercepções, os indivíduos que perceberam seus parceiros como andróginos relataram níveis mais elevados de satisfação na relação do que os outros tipos.

A qualidade da relação romântica é um fator importante para o bem-estar dos envolvidos. Estudos com diferentes tipos de díades mostraram que o relacionamento entre casais do mesmo sexo é semelhante ao de casais heterossexuais (Antonelli, Dèttore, Lasagni, Snyder, \& Balderrama-Durbin, 2014; Cusack, Hughes, \& Cook, 2012; Frost \& Gola, 2015; Gottman et al., 2003; Graham \& Barnow, 2013; Kurdek, 2006; Means-Christensen et al., 2003; Peplau \& Fingerhut, 2007). $\mathrm{Na}$ presente pesquisa, os gays e lésbicas mostraram-se 
mais satisfeitos do que os indivíduos heterossexuais, contudo, esse efeito foi muito pequeno. Poucos estudos encontrados sugeriram que as lésbicas seriam melhores na comunicação e mais satisfeitas em seus relacionamentos românticos do que as mulheres heterossexuais (Kurdek, 2008). Corroborando, Meuwly, Feinstein, Davila, Nuñez e Bodenmann (2013) registraram que as lésbicas relataram mais apoio, menos conflito e mais satisfação com as suas parceiras do que as mulheres heterossexuais. Rostosky e Riggle (2017) realizaram uma revisão de 66 estudos empíricos de amostras norte-americanas sobre relações de casais do mesmo sexo publicadas de 2000 a 2016. Os resultados revelaram os seguintes pontos: processos de relacionamento (respeito e valorização das diferenças individuais, emoções e interações positivas, comunicação e negociação eficazes) e características positivas de relacionamento (intimidade, compromisso e ideais igualitários).

Em geral, os presentes achados sugerem que argumentos para a negação de igualdade de acesso à vida conjugal, baseados em suposições de diferenças no significado social e psicológico do amor romântico entre casais homossexuais e heterossexuais, não tiveram apoio nesses dados empíricos atuais. Os componentes do amor, principalmente a combinação de Intimidade e Decisão/Compromisso, com pequenas variações, revelaram-se responsáveis pela Satisfação Conjugal de gays, lésbicas e heterossexuais, indicando que, possivelmente, é o que mais importou nessas interações amorosas, independentemente das Orientações Sexuais dessas pessoas.

Contudo, é preciso considerar que as amostras desta pesquisa são do tipo de conveniência e podem não representar adequadamente essas populações examinadas do Rio de Janeiro/RJ. Sugere-se, também, que novas investidas com escores de pessoas de outras regiões brasileiras poderão avançar na tarefa de gerar evidências mais abrangentes e contribuir para o estudo dessa importante questão social.

\section{Referências}

Antonelli, P., Dèttore, D., Lasagni, I., Snyder, D. K., \& Balderrama-Durbin, C. (2014). Gay and lesbian couples in Italy: Comparisons with heterosexual couples. Family Process, 53(4), 702-716. doi: 10.1111/famp. 12078

Arbuckle, J. L. (2014). IBM® SPSS® Amos ${ }^{\mathrm{TM}} 23$ User's Guide. Chicago: SPSS.
Bailey, W. C., Hendrick, C., \& Hendrick, S. S. (1987). Relation of sex and gender role to love, sexual attitudes, and self-esteem. Sex Roles, 16, 637-648. doi: 10.1007/BF00300378

Bauermeister, J. A., Johns, M. M., Pingel, E., Eisenberg, A., Santana, M. L., \& Zimmerman, M. (2011). Measuring love: Sexual minority male youths' ideal romantic characteristics. Journal of LGBT Issues in Counseling, 5(2), 102-121. doi: 10.1080/15538605.2011.574573

Bem, S. L. (1974). The measurement of psychological androgyny. Journal of Consulting and Clinical Psychology, 42, 155-162. doi:10.1037/h0036215

Bergamo, M. (2016). IBGE pretende fazer primeira pesquisa nacional sobre população LGBT. Folha de São Paulo. Recuperado de http://www1.folha. uol.com.br/colunas/monicabergamo/2016/12/ 1838027-ibge- pretende-fazer-primeira-pesquisa-nacional-sobre-populacao-lgbt.shtml

Blashill, A. J., \& Powlishta, K. K. (2009). Gay stereotypes: The use of sexual orientation as a cue for gender-related attributes. Sex Roles, 61, 783-793. doi: 10.1007/s11199-009-9684-7

Burn, S. M., \& Ward, A. Z. (2005). Men's conformity to traditional masculinity and relationship satisfaction. Psychology of Men \& Masculinity, 6, 254-263. doi: 10.1037/1524-9220.6.4.254

Byrne, B. M. (2016). Structural equation modeling with amos: Basic concepts, applications, and programming. New York: Routledge.

Curun, F. (2012). Sex role orientation and romantic relationship satisfaction: A study in Turkey. Interpersona, 6, 227-234. doi: 10.5964/ijpr.v6i2.103

Cusack, C. E., Hughes, J. L., \& Cook, R. E. (2012). Components of love and relationship satisfaction: Lesbians and heterosexual women. Psi Chi Journal of Psychological Research, 17(4), 171-179. doi:10.24839/2164-8204.JN17.4.171

Damásio, B. (2013). Contribuições da análise fatorial confirmatória multigrupo (AFCMG) na avaliação de invariância de instrumentos psicométricos. Psico-USF, 18(2), p. 211-220. doi: 10.1590/ S1413-82712013000200005

Dean, M. L., \& Tate, C. C. (2017). Extending the legacy of sandra bem: Psychological androgyny as a touchstone conceptual advance for the study of 
gender in psychological science. Sex Roles, 76(1112), 643-654. doi: 10.1007/s11199-016-0713-z

Deenen, A. A., Gijs, L., \& van Naerssen, A. X. (1994). Intimacy and sexuality in gay male couples. Archives of Sexual Behavior, 23(4), 421-431. doi: 10.1007/ BF01541407

DiBennardo, R., \& Gates, G. J. (2014). Research note: US census same-sex couple data: adjustments to reduce measurement error and empirical implications. Population Research and Policy Review, 33, 603-614. doi: 10.1007/s11113-013-9289-2

Doyle, D. M., \& Molix, L. (2015). Social stigma and sexual minorities' romantic relationship functioning: A meta-analytic review. Personality and Social Psychology Bulletin, 41(10) 1363-1381. doi: 10.1177/0146167215594592

Farias, M. de O. (2010). Mitos atribuídos às pessoas homossexuais e o preconceito em relação à conjugalidade homossexual e a homoparentalidade. Revista de Psicologia da UNESP, 9(1), 104-115. Recuperado de http://seer.assis.unesp.br/index.php/ psicologia/article/view/431/410

Finney, S. J., \& DiStefano, C. (2006). Non-normal and categorical data in structural equation modeling. Em G. R. Hancock \& R. O. Mueller (Orgs.), Structural equation modeling: $A$ second course (pp. 269-314). Greenwich, CT: Information Age Publishing.

Fornell, C., \& Larcker, D. F. (1981). Evaluating structural equation models with unobservable variables and measurement error. Journal of Marketing Research, 18(1), 39-50. doi: 10.2307/3151312

Frost, D. M., \& LeBlanc, A. J. (2014). Nonevent stress contributes to mental health disparities based on sexual orientation: Evidence from a personal projects analysis. American Journal of Orthopsychiatry, 84, 557-566. doi: 10.1037/ort0000024

Frost, D. M., \& Gola, K. A. (2015). Meanings of intimacy: A comparison of members of heterosexual and same-sex couples. Analyses of Social Issues and Public Policy, 15, 382- 400. doi:10.1111/asap.12072

Gates, G. J., \& Newport, F. (2015). An estimated 780,000 Americans in same-sex marriages. Gallup Social Issues. Recuperado de http://www.gallup. com/poll/182837/estimated-780-000-americans-sex-marriages.aspx
Gouveia, V. V., Fonseca, P. N. da, Cavalcanti, J. P. N., Diniz, P. K. da C., \& Dória, L. C. (2009). Versão abreviada da Escala Triangular do Amor: Evidências de validade fatorial e consistência interna. Estudos de Psicologia, 14, 31-39. doi: 10.1590/ S1413-294X2009000100005

Gottman, J. M., Levenson, R. W., Gross, J., Frederickson, B. L., McCoy, K., Rosenthal, L.,... Yoshimoto, D. (2003). Correlates of gay and lesbian couples' relationship satisfaction and relationship dissolution. Journal of Homosexuality, 45, 23-43. doi: 10.1300/J082v45n01_02

Graham, J. M., \& Barnow, Z. B. (2013). Stress and social support in gay, lesbian, and heterosexual couples: Direct effects and buffering models. Journal of Family Psychology, 27, 569 -578. doi:10.1037/a0033420

Hair, J. F., Black, W. C., Babin, B. J., \& Anderson, R. E. (2014). Multivariate Data Analysis (7 $7^{\mathrm{a}} \mathrm{Ed}$.). Harlow, UK: Pearson.

Hendrick, S. S. (1988). A generic measure of relationship satisfaction. Journal of Marriage and the family, 50, 93-98. doi:10.2307/352430

Hernandez, J. A. E. (2009). Reavaliando o bem sex-role inventory. Estudos de Psicologia, 26, 73-83. doi:10.1590/S0103-166X2009000100008

Hernandez, J. A. E. (2014). Evidências de validade da Escala de Avaliação do Relacionamento. Estudos de Psicologia, 31, 327-336. doi:10.1590/0103-166X2014000300001

Hernandez, J. A. E. (2016). Análise fatorial exploratória e hierárquica da Escala Triangular do Amor. Avaliação Psicológica, 15(1), 11-20. doi:10.15689/ ap.2016.1501.02

Hernandez, J. A. E., \& Soares (2013). Gênero, amor e satisfação em casais homo e heterossexuais. Em A. Garcia \& J. Fitzpatrick (Orgs.), Relações Românticas, Conjugais e Parassociais (pp. 68-88). Vitória: UFES.

Jackson, J. B., Miller, R. B., Oka, M., \& Henry, R. G. (2014). Gender differences in marital satisfaction: A meta-analysis. Journal of Marriage and Family, 76, 105-129. doi:10.1111/jomf.12077

Kachel, S., Steffens, M. C., \& Niedlich, C. (2016). Traditional masculinity and femininity: Validation of a new scale assessing gender roles. Frontiers in Psychology, 7, 956. doi: 10.3389/fpsyg.2016.00956 
Kochar, R. K., \& Sharma, D. (2015). Role of love in relationship satisfaction. The International Journal of Indian Psychology, 3, Issue 1, $\mathrm{n}^{\circ}$ 6. Recuperado de http://oaji.net/articles/2015/1170-1447398407. pdf

Kurdek, L. A. (2006). Differences between partners from heterosexual, gay, and lesbian cohabiting couples. Journal of Marriage and Family, 68(2), 509528. doi:10.1111/j.1741-3737.2006.00268.x

Kurdek, L. A. (2008). Change in relationship quality for partners from lesbian, gay male, and heterosexual couples. Journal of Family Psychology, 22(5), 701-711. doi: 10.1037/0893-3200.22.5.701

Lafontaine, M. F., Gabbay, N., Péloquin, K., Flesch, J. L., \& Fitzpatrick, J. (2013). An overview of samesex couples' love lives. Integrating Science \& Practice, 3(2), 18-21. Recuperado de https://www.ordrepsy. qc.ca/pdf/2013_11_01_Integrating_SandP_Dossier_04_Lafontaine.pdf

Lavner, J. A. (2017). Relationship satisfaction in lesbian couples: Review, methodological critique, and research agenda. Journal of Lesbian Studies, 21(1), 729. doi:10.1080/10894160.2016.1142348

Lewis, D. M. (2011). Intimacy, passion, and commitment as predictors of couples' relationship satisfaction. Dissertation and Theses- Gradworks, 171 pages. Recuperado de http:/ / search.proquest.com/docview /894264700?accountid $=12045$

Liben, L. S., \& Bigler, R. S. (2017). Understanding and undermining the development of gender dichotomies: The legacy of sandra lipsitz bem. Advance online publication in Sex Roles, 76(9-10), 544-555. doi: 10.1007/s11199-015-0519-4

Marôco, J. (2014). Análise de equações estruturais. Fundamentos teóricos, software \& aplicaçoes. Pêro Pinheiro: ReportNumber.

Means-Christensen, A. J., Snyder, D. K., \& Negy, C. (2003). Assessing nontraditional couples: Validity of The Marital Satisfaction Inventory - Revised with gay, lesbian, and cohabiting heterosexual couples. Journal of Marital and Family Therapy, 29, 69-83. doi:10.1111/j.1752-0606.2003.tb00384.x

Meuwly, N., Feinstein, B. A., Davila, J., Nuñez, D. G., \& Bodenmann, G. (2013). Relationship quality among swiss women in opposite-sex versus same-sex romantic relationships. Swiss Journal of Psychology, 72(4), 2013, 229-233. doi: 10.1024/1421-0185/ a000115

Peplau, A. L., \& Fingerhut, A. W. (2007). The close relationships of lesbians and gay men. Annual Review of Psychology, 58, 405-424. doi:10.1146/annurev. psych.58.110405.085701

Rostami, A., Ghazinour, M., Nygren, L., \& Richter, J. (2014). Marital satisfaction with a special focus on gender differences in medical staff in tehran, Iran. Journal of Family Issues, 35(14) 1940-1958. doi: 10.1177/0192513X13483292

Rostosky, S. S., \& Riggle, E. D. B. (2017). Same-sex couple relationship strengths: A review and synthesis of the empirical literature (2000-2016). Psychology of Sexual Orientation and Gender Diversity. Advance online publication. doi: 10.1037/ $\operatorname{sgd} 0000216$

Starr, C. R., \& Zurbriggen, E. L. (2016). Sandra bem's gender schema theory after 34 years: A review of its reach and impact. Sex Roles, 76(9-10), 566-578. doi: 10.1007/s11199-016-0591-4

Sternberg, R. J. (1997). A construct-validation of a triangular love scale. European Journal of Social Psychology, 27, 313-335. doi: 10.1002/ (SICI)1099-0992(199705)27:33.3.CO;2-W

Sternberg, R. J. (2006). A duplex theory of love. Em R. J. Sternberg \& K. Weis (Orgs.), The New Psychology of Love (pp. 184-199). New Haven: Yale University Press.

Toledo, L. G., \& Teixeira Filho, F. S. (2012). As lesbianidades entre o estigma da promiscuidade e da ilegitimidade sexual. Temáticas, 20(40), 67-103. Recuperado de https://www.ifch.unicamp.br/ojs/ index.php/tematicas/article/view/2206/1612

Yela, C. (1998). Temporal course of the basic components of love throughout relationships. Psychology in Spain, 2(1), 76-86. Recuperado de http:/ /www.psychologyinspain.com/content/reprints/1998/9.pdf

Recebido em: 06/02/2018

Reformulado em: 03/01/2019

Aceito: 21/01/2019 
Nota dos autores:

Esta pesquisa foi financiada pelo programa APQ1 da Fundação Carlos Chagas Filho de Amparo à Pesquisa do Estado do Rio de Janeiro (FAPERJ).

Sobre os autores:

José Augusto Evangelho Hernandez é psicólogo, mestre e doutor em Psicologia do Desenvolvimento (UFRGS). Atualmente é professor do Programa de Pós-graduação em Psicologia Social da Universidade do Estado do Rio de Janeiro (UERJ) e desenvolve atividades de ensino e pesquisa em relacionamentos íntimos, gênero e construção e adaptação de medidas psicológicas e coordena o Núcleo de Mensuração em Psicologia (NUMERA).

ORCID: http://orcid.org/0000-0001-9402-7535

E-mail: hernandez.uerj@gmail.com

Vera Lucia da Annunciação Baylão Soares é psicóloga pela Universidade do Estado do Rio de Janeiro (UERJ). Atualmente dedica-se à clínica psicológica, à pós-graduacão em

Gestalt-Terapia pela Pontifícia Universidade Católica do Rio de Janeiro e à graduação em Letras pela Universidade Federal Fluminense.

ORCID: https://orcid.org/0000-0002-0707-1544

E-mail:verabaylao@yahoo.com.br

Contato com os autores:

Instituto de Psicologia

Universidade do Estado do Rio de Janeiro

Rua São Francisco Xavier, 514 - $10^{\circ}$ andar - sala 10028D, Maracanã

Rio de Janeiro-RJ, Brasil

CEP: 20550-900 